\title{
Mesure d'écoulement du contenu réticulaire de vache
}

\author{
C Dardillat \\ Station de Recherches sur la Nutrition des Herbivores, \\ INRA, Theix, 63122 Ceyrat, France
}

Le contenu réticulaire (CR), bien que plus fluide que ceux des autres pré-estomacs, ne s'écoule pas aisément à travers un trou de la taille de l'orifice réticulo-omasal (10 $\mathrm{x}$ $45 \mathrm{~mm}$, McBride et al, 1983). Après passage de quelques $\mathrm{ml}$ de CR partiellement filtré, l'occlusion survient (Dardillat et Baumont, 1988). II est donc important de pouvoir caractériser cette aptitude à s'écouler "flowability" dans les études de transit digestif.

Cette filtration-occlusion est la résultante de la différence de vitesse d'écoulement entre la phase liquide et les particules. Ces dernières, de formes irrégulières et très proches les unes des autres, sont mutuellement freinées par frottement contre la paroi. Au contraire, la phase liquide dont la viscosité $(0,8$ à $1 \mathrm{mPa} / \mathrm{s}$ à $39^{\circ} \mathrm{C}$ ) est proche de celle de l'eau $(0,7)$, s'écoule aisément. Ce comportement particulier ne peut être traduit par une mesure avec un viscosimètre. La méthode que nous proposons simule le phénomène et consiste simplement à mesurer le diamètre de l'orifice le plus petit par lequel le CR s'écoule en totalité. Ce même type de mesure a d'ailleurs été proposé par Gioia (1980) pour caractériser l'écoulement des poudres. Le matériel que nous avons mis au point consiste en 2 boîtes de 11 et une série de couvercles en matière plastique de $10 \times 10 \mathrm{~cm}$ que nous avons percés de 2 trous dans 2 angles opposés et dont la taille s'échelonne de 14 à 50 $\mathrm{mm}$ par intervalle de $2 \mathrm{~mm}$. Sur du CR qui vient d'être prélevé, les essais répétés de vidage d'une boîte dans l'autre, avec différents couvercles pour déterminer la taille minimale du trou qui convient, demandent moins de $2 \mathrm{~min}$. Dans ces conditions, une agitation continue et une thermostatisation sont inutiles (un écart de température de $10^{\circ} \mathrm{C}$ entraîne une erreur de $2 \mathrm{~mm}$ ).

Les essais effectués montrent des index d'écoulement allant de 16 à $44 \mathrm{~mm}$; la richesse en grandes particules et le taux de MS sont les deux facteurs de variation essentiels de cet index. Les différences d'un jour à l'autre sont plus importantes qu'entre régime. Ainsi l'herbe fraîche et les granulés de luzerne entraînent un même index de $30 \mathrm{~mm}$ parce que l'absence de fibres longues avec les granulés est compensée par un taux de MS élevé : $11,8 \%$ au lieu de $3,5 \%$. Nous étudions actuellement comment varie cet index avec la prise alimentaire, pour différents régimes.

Dardillat C, Baumont R (1988) Reprod Nutr Dev 28 (suppl 1), 137-138

Gioia A (1980) Pharm Technol February, revue interne Bioblock Scientific, F-67403, llikirch

McBride BW, Milligan LP, Turner BV (1983) $J$ Agric Sci Cambridge 101, 749-750 